\section{Comparison of ultrasound biomicroscopic parameters after laser iridotomy in eyes with primary angle closure and primary angle closure glaucoma}

T Dada', S Mohan', R Sihota', R Gupta', V Gupta ${ }^{1}$ and RM Pandey²

\begin{abstract}
${ }^{1}$ Glaucoma Research Laboratory, Dr Rajendra Prasad Centre for Ophthalmic Sciences, All India Institute of Medical Sciences, New Delhi, India
\end{abstract}

${ }^{2}$ Department of Biostatistics, All India Institute of Medical Sciences, New Delhi, India

Correspondence: T Dada, Dr Rajendra Prasad Centre for Ophthalmic Sciences, All India Institute of Medical Sciences, Ansari Nagar, New Delhi 110029, India Tel: + 91011 26589695; Fax: + 9101126588919 . E-mail: tanujdada@ hotmail.com

Received: 11 August 2005 Accepted in revised form: 19 March 2006 Published online: 5 May 2006

\begin{abstract}
Purpose To study changes in anterior segment morphology after laser peripheral iridotomy (LPI) in primary angle closure (PAC) and primary angle closure glaucoma (PACG) using ultrasound biomicroscopy (UBM).

Methods Ninety-three eyes of 93 patients underwent anterior segment evaluation including gonioscopy, disc evaluation with + 90D lens, applanation intraocular pressure, and standard achromatic perimetry. UBM was performed before and 2 weeks after Nd:YAG LPI to measure the trabecular-iris angle (TIA), the angle-opening distance (AOD 250/500), and the central anterior chamber depth (ACD). Results The superior TIA widened from a mean of $7.54 \pm 3.15$ to $15.66 \pm 6.69^{\circ}(P=0.0001)$, the inferior TIA increased from a mean of $9.0 \pm 4.7$ to $15.9 \pm 6.8^{\circ}(P=0.0001)$ after LPI in PAC. In PACG, the mean superior angle changed from $4.55 \pm 2.5$ to $6.12 \pm 3.8^{\circ}(P=0.4)$ and the inferior angle increased from $4.75 \pm 2.0$ to $7.9 \pm 3.7^{\circ}(P=0.1)$. The mean ACD increased from $2.19 \pm 0.36$ to $2.30 \pm 0.36 \mathrm{~mm}$ in PAC group $(P=0.0003)$, with no significant change seen in the PACG group $(1.79 \pm 0.32$ vs $1.82 \pm 0.33 \mathrm{~mm}$, $P=\mathbf{0 . 1 3}$ ).

Conclusion LPI leads to a widening of the anterior chamber angle and a deepening of the anterior chamber in eyes with PAC. It does not significantly change any anterior segment parameters in eyes with PACG. Eye (2007) 21, 956-961; doi:10.1038/sj.eye.6702360; published online 5 May 2006
\end{abstract}

Keywords: ultrasound biomicroscopy; laser iridotomy; primary angle closure; primary angle closure glaucoma

Primary angle-closure glaucoma (PACG) is the major form of glaucoma in the Asian population and an important cause for blindness worldwide. ${ }^{1}$ The development of primary angle closure (PAC) into PACG can be prevented by performing a laser peripheral iridotomy (LPI). ${ }^{2-5}$ This is especially useful in preventing recurrence of acute attacks and eliminating the risk of an acute attack in eyes with intermittent angle closure or predisposed fellow eyes. ${ }^{6,7}$ In eyes with PAC, an LPI may help in reversing appositional angle closure and control the intraocular pressure (IOP). ${ }^{8,9}$ The mechanism of action of an LPI is that it creates an opening to bypass the pupillary block and thereby allows a convex iris to flatten and widen the anterior chamber angle. ${ }^{10,11}$

However, there has been no accurate and objective documentation of the changes in anterior chamber angle morphology induced by an LPI in various subtypes of PACG.

Gonioscopic studies are limited by observer bias and do not allow for an accurate estimation of the angle recess. Ultrasound biomicroscopy (UBM) is a high-resolution imaging of the anterior segment that allows an objective evaluation of the angle morphology using an automated software programme. ${ }^{12-14}$ In the present study we undertook a prospective evaluation of the changes in the anterior segment morphology induced by an LPI in eyes 
with PACG and tried to find out the difference between PAC and PACG, following LPI.

\section{Materials and methods}

Consecutive patients with PAC and PACG attending the glaucoma service of a tertiary care eye centre were included in the present study. The diagnosis was based on clinical history, slit lamp and ophthalmoscopic examinations, gonioscopy, IOP measurement, and visual field testing. The diagnosis of various subtypes of PACG were established in the following manner:

\section{Primary angle closure}

Patients with PAC had to have gonioscopically documented presence of peripheral anterior synechiae, clumping of pigment in an occludable angle that is one with a steep peripheral iris configuration and an angle recess narrowed to less than $20^{\circ}$, with the trabecular meshwork not being visualized in more than $180^{\circ}$ of the angle. The optic disc did not have glaucomatous damage.

\section{Primary angle closure glaucoma}

These patients had a chronically elevated IOP (>21 mmHg), gonioscopically confirmed peripheral anterior synechiae of more than $180^{\circ}$, and optic nerve head and visual field changes typical of glaucoma.

All cases underwent a detailed eye examination including measurement of visual acuity, slit-lamp biomicroscopy, direct ophthalmoscopy, $\pm 90 \mathrm{D}$ fundus examination, gonioscopy, applanation tonometry, visual field testing (Humphrey 30-2 test), A-scan biometry, and UBM. Pilocarpine was stopped atleast 1 week before UBM measurements and patients were put on oral acetazlamide ( $250 \mathrm{mg}$ TDS) and $0.5 \%$ timolol eye drops.

UBM was performed on the UBM Model 840 machine (Paradigm medical Industries, Inc, USA) with a $50-\mathrm{MHz}$ transducer probe. After topical anaesthesia with $0.5 \%$ proparacaine, the eyes were studied using an eyecup, filled with $2 \%$ methylcellulose as a coupling agent, with care taken not to exert pressure on the globe. Variation in accommodation was minimized by fixation with the contralateral eye on a standard distance target on the ceiling. Each eye was examined in its axial section with the probe kept perpendicular to the corneo-scleral surface in constant ambient lighting condition. Three images showing the superior and inferior anterior chamber angle and ciliary body were frozen and recorded for each eye. Areas with peripheral anterior synechiae formation and a completely closed angle were avoided and only images with a visible angle recess were included for measurements. An image centred on the pupil was also captured. The various anterior segment parameters (Figure 1), listed below, were measured on these images using a caliper incorporated within the instrument's software package. The procedure was repeated 2 weeks after performing an LPI. The UBM images pre- and post-LPI were taken by a single trained ophthalmologist (TD) and the post-LPI image saved for measurement did not show the iridotomy site. The measurements were performed by another ophthalmologist (SM) who was masked to whether the image was taken before or after iridotomy. It is important to mask the person taking the UBM measurements to the diagnosis as this is a manual procedure which can introduce bias into the study.

1. The trabeculer-iris angle (TIA) measured with its apex in the iris recess and the arms of the angle passing through a point on the trabecular meshwork at $500 \mu \mathrm{m}$ from the scleral spur and the point on the iris perpendicularly opposite.

2. The trabecular-ciliary process distance (TCPD), measured on a line extending from the corneal endothelium at $500 \mu \mathrm{m}$ from the scleral spur perpendicularly through the iris to the ciliary processes.

3. The AOD 500 is the distance between the posterior corneal surface and the anterior iris surface measured on a line perpendicular to the trabecular meshwork at $500 \mu \mathrm{m}$ from the scleral spur.

4. The AOD 250 is the distance between the posterior corneal surface and the anterior iris surface measured on a line perpendicular to the trabecular meshwork at $250 \mu \mathrm{m}$ from the scleral spur.

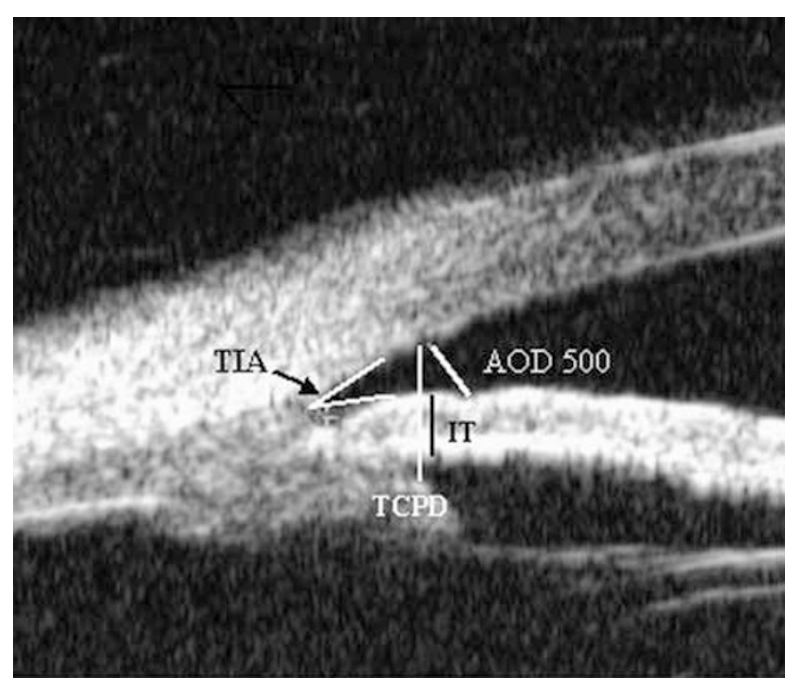

Figure 1 Measurements of anterior segment parameters on UBM. 
5. The ICPD is the distance measured from the posterior iris surface (iris pigmented epithelium to the ciliary process along the same line as the TCPD.

6. The ILCD, measured along the iris pigmented epithelium from the pupillary border to the point where the anterior lens surface leaves the iris.

7. The ILA corresponds to the angle between the iris and the lens near the pupillary edge.

8. IT, measured by a perpendicular drawn at $500 \mu \mathrm{m}$ from the scleral spur.

9. The SS-IR, measured by the UBM pro software directly along a perpendicular from the scleral spur to the iris

10. Central ACD was measured by a perpendicular drawn from the posterior corneal surface up to a horizontal line parallel to the edge of the pupil.

The LPI was performed using a Nd:YAG (neodymium-yttrium-aluminium-garnet) laser set at 2-6 mJ. A crypt in the midperipheral iris was selected for the iridotomy site. All patients received $0.1 \%$ betamethasone eye drops (q.i.d.) for 1 week in addition to the anti-glaucoma medications they were already receiving (excluding pilocarpine). Patients with PAC were given $0.5 \%$ timolol eye drops (b.i.d.) for a 1-week period after the LPI.

Statistical analysis was performed using STATA version 8.0 programme and a $P$-value of $<0.5$ was taken as statistically significant. Wilcoxon's-Sign-Rank test and $t$-test were used to analyse the UBM parameters.

\section{Results}

Ninety-three eyes of 93 patients with PAC or PACG were enrolled in the present study. The mean age was $48.08 \pm 9.99$ years (range: $30-76$ years). There were 24 males and 69 females. Fifty-four eyes had PAC whereas 39 eyes had PACG. The mean age of the PAC group was $47.3 \pm 6.46$ years and the mean age of the PACG group was $48.7 \pm 5.42$ years $(P=0.12)$.

After LPI, UBM anterior chamber angle recording revealed that the mean superior TIA increased significantly from $6.46 \pm 6.68$ to $12.1 \pm 8.1^{\circ}$, and mean inferior TIA increased from $7.5 \pm 6.7$ to $12.9 \pm 8.5^{\circ}$ $(P=0.0001)$. Superior AOD250 increased from $0.039 \pm 0.059$ to $0.080 \pm 0.09 \mathrm{~mm}$ and AOD500 from $0.095 \pm 0.09$ to $0.17 \pm 0.133 \mathrm{~mm}(P=0.0001)$. The inferior AOD250 and AOD500 increased from $0.049 \pm 0.05$ to $0.131 \pm 0.22 \mathrm{~mm}$ and $0.125 \pm 0.24$ to $0.230 \pm 0.12 \mathrm{~mm}$ $(P=0.0001)$, respectively.

There was no significant change in the trabecular ciliary process distance $(0.751 \pm 0.22$ to $0.785 \pm 0.23 \mathrm{~mm}$, $P=0.12)$, the ICPD $(0.479 \pm 0.10$ to $0.474 \pm 0.10 \mathrm{~mm}$, $P=0.3)$ or the IT $(0.192 \pm 0.16$ to $0.186 \pm 0.15 \mathrm{~mm}$
$(P=0.41)$. The mean ILCD increased from $1.208 \pm 0.35$ to $1.33 \pm 0.46 \mathrm{~mm}$ whereas the mean ILA increased from $20.4 \pm 83.4$ to $21.1 \pm 94.1^{\circ}$ after iridotomy (NS). There was no significant change in the mean $\mathrm{AC}$ depth before and after the iridotomy $(2.05 \pm 0.39$ to $2.16 \pm 0.39 \mathrm{~mm})$.

A subgroup analysis was then performed to evaluate the changes in eyes with PAC vs eyes with PACG. Table 1 shows changes as seen in PAC and Table 2 shows the changes in PACG with their statistical significance. There was significant widening of the superior and inferior anterior chamber angle along with an increase in the AODs in PAC (Figure 2a and b) but not in PACG. There was significant increase in the trabecular ciliary process distance and the scleral spur to iris distance after LPI in PAC with no significant change in PACG. There was a decrease in the ILCD after LPI in PAC group. The mean ILA, IT, and iris ciliary process distance showed no significant change after laser iridotomy in either group. There was no significant change in the mean AC depth

Table 1 Change in UBM parameters in PAC after LPI

\begin{tabular}{lccl}
\hline UBM parameter & Pre-LPI (mean) & Post-LPI (mean) & P-value \\
\hline Sup TIA (deg) & $7.54 \pm 3.15$ & $15.66 \pm 6.69$ & 0.0001 \\
AOD250 (mm) & $0.048 \pm 0.06$ & $0.109 \pm 0.10$ & 0.0001 \\
AOD500 (mm) & $0.107 \pm 0.09$ & $0.208 \pm 0.18$ & 0.0001 \\
TCPD (mm) & $0.748 \pm 0.19$ & $0.837 \pm 0.19$ & 0.0001 \\
IT (mm) & $0.473 \pm 0.08$ & $0.486 \pm 0.11$ & 0.42 \\
ICPD (mm) & $0.169 \pm 0.15$ & $0.204 \pm 0.136$ & 0.23 \\
ILCD (mm) & $1.220 \pm 0.32$ & $1.166 \pm 0.32$ & 0.0174 \\
ILA (mm) & $26.3 \pm 104$ & $28.1 \pm 116$ & 0.21 \\
INF TIA (deg) & $9.0 \pm 4.7$ & $15.9 \pm 6.8$ & 0.0001 \\
AODI250 (mm) & $0.059 \pm 0.061$ & $0.178 \pm 0.026$ & 0.0001 \\
AODI500 (mm) & $0.150 \pm 0.012$ & $0.291 \pm 0.26$ & 0.0001 \\
ACD (mm) & $2.19 \pm 0.36$ & $2.30 \pm 0.36$ & 0.0003 \\
SSIR (mm) & $0.256 \pm 0.27$ & $0.396 \pm 0.15$ & 0.001 \\
ARA (mm $\left.{ }^{2}\right)$ & $0.132 \pm 0.19$ & $0.158 \pm 0.16$ & 0.0207 \\
\hline
\end{tabular}

Table 2 Changes in UBM parameters in PACG after LPI $(n=39)$

\begin{tabular}{lccc}
\hline UBM parameter & Pre-LPI & Post-LPI & P-value \\
\hline Sup TIA (deg) & $4.55 \pm 2.5$ & $6.12 \pm 3.8$ & 0.403 \\
AOD250 (mm) & $0.025 \pm 0.043$ & $0.032 \pm 0.043$ & 0.518 \\
AOD500 (mm) & $0.065 \pm 0.09$ & $0.076 \pm 0.13$ & 0.273 \\
TCPD (mm) & $0.756 \pm 0.26$ & $0.689 \pm 0.26$ & 0.343 \\
IT (mm) & $0.492 \pm 0.12$ & $0.484 \pm 0.096$ & 0.195 \\
ICPD (mm) & $0.233 \pm 0.17$ & $0.253 \pm 0.16$ & 0.087 \\
ILCD (mm) & $1.181 \pm 0.42$ & $1.26 \pm 0.51$ & 0.08 \\
ILA (degr) & $9.9 \pm 4.1$ & $8.1 \pm 3.8$ & 0.110 \\
INF TIA (deg) & $4.75 \pm 2.0$ & $7.9 \pm 3.7$ & 0.109 \\
AODI250 (mm) & $0.031 \pm 0.048$ & $0.054 \pm 0.08$ & 0.210 \\
AODI500 (mm) & $0.081 \pm 0.09$ & $0.160 \pm 0.17$ & 0.042 \\
ACD (mm) & $1.79 \pm 0.32$ & $1.82 \pm 0.33$ & 0.131 \\
SSIR (mm) & $0.406 \pm 0.24$ & $0.305 \pm 0.22$ & 0.145 \\
ARA $\left(\mathrm{mm}{ }^{2}\right)$ & $0.047 \pm 0.07$ & $0.0792 \pm 0.06$ & 0.104 \\
\hline
\end{tabular}



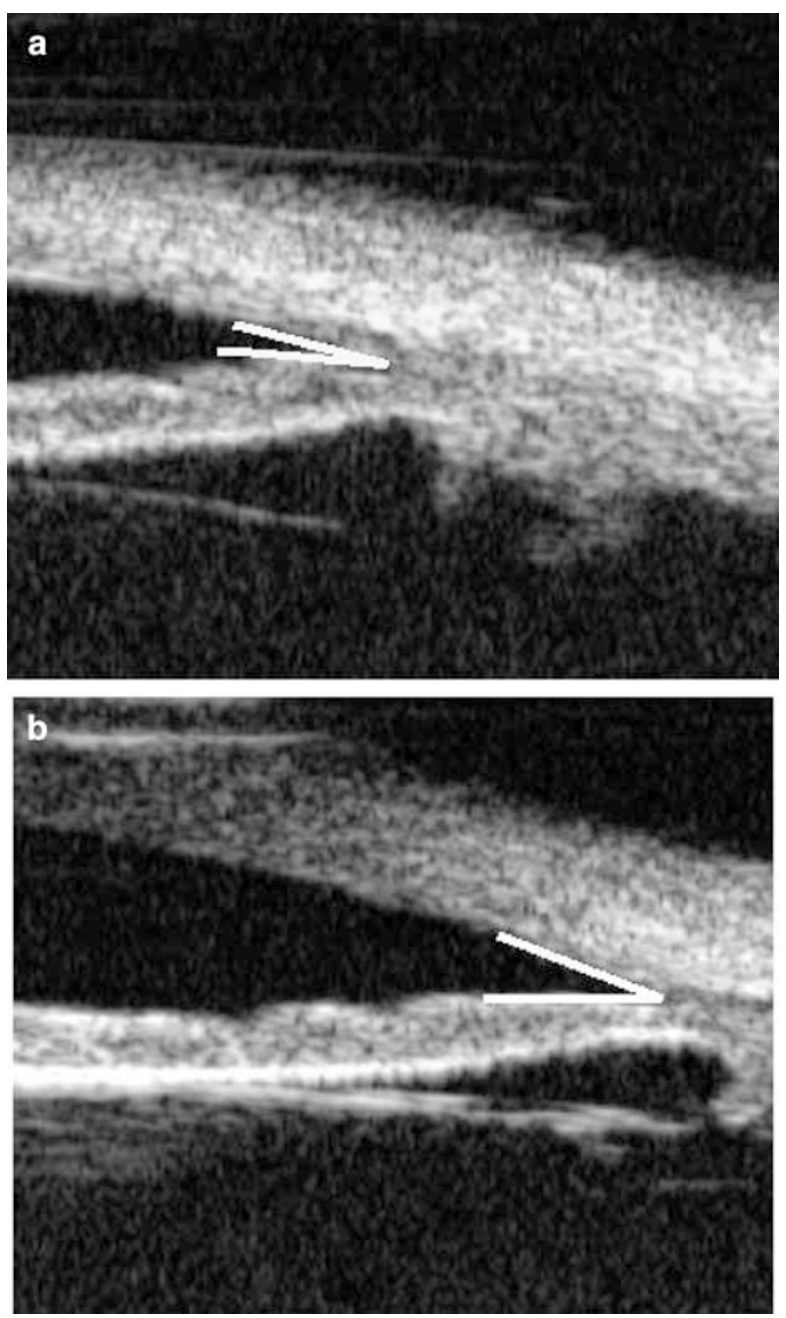

Figure 2 (a) Narrow angle recess in PAC. (b) Widening of angle recess after laser iridotomy in PAC.

before and after the iridotomy in PACG but there was a deepening of the anterior chamber noted in PAC.

\section{Discussion}

Although Nd:YAG iridotomy is now used as the standard treatment in eyes with PACG, very few studies have been performed to document the effect of doing an LPI in these eyes. The mean age of patients in the present study was less than 50 years, which reflects a racial predilection at a much younger age in our population as is evident from other studies on PACG conducted by us. $^{15,16}$

To date no study has reported the effect of LPI on PAC and PACG patients by using UBM. The present study is the first one to demonstrate clearly that there is a widening of the anterior chamber angle and deepening of the anterior chamber after LPI in eyes with PAC but there is no significant change in any of the anterior segment parameters in eyes with PACG. The main cause for this is the closure of the angle by extensive peripheral anterior synechiae in PACG. The superior angle widened from a baseline value of 7.5 to $15.6^{\circ}$, whereas the inferior angular width increased from 9 to $15.9^{\circ}$ in eyes with PAC as a consequence of the laser iridotomy. The AOD500 increased from 0.1 to $0.2 \mathrm{~mm}$ for the superior angle and from 0.08 to $0.16 \mathrm{~mm}$ for the inferior angle.

Gazzard et $a l^{17}$ studied the effect of LPI on 55 fellow eyes of acute PACG patients. The AOD250, AOD500, and angle recess area all significantly increased after sequential laser iridotomy but the ACD did not change significantly (2.41 vs $2.42 \mathrm{~mm}$ ) as measured with optical pachymetry. The authors concluded that in Asian eyes at high risk of developing acute PAC, LPI produced a significant widening of the anterior chamber angle without deepening the anterior chamber centrally. Yoon et $\mathrm{al}^{18}$ reported a statistically significant increases in AOD500, TIA, and ILCD in 13 eyes with PACG. The ACD, TCPD, and IT were not changed significantly in PACG. The changes in angle configuration after LPI was reported to be due to alterations in aqueous pressure gradients across the iris.

We found that mean ILCD decreased from 1.22 to $1.166 \pm 0.32 \mathrm{~mm}(P=0.0174)$ in PAC and did not change significantly in the PACG group. Caronia et al ${ }^{10}$ prospectively performed UBM and A-scan biometry in 13 eyes of 13 consecutive untreated patients with relative pupillary block and appositional angle closure, without peripheral anterior synechiae on indentation gonioscopy. UBM was performed before and 1 week after laser iridotomy in each eye. The AOD increased from a mean value of 0.11 to $0.18 \mathrm{~mm}$ and the mean trabecular iris angle from 8.3 to $18.6^{\circ}$. In this study, the mean ILCD increased from 0.58 to $1.18 \mathrm{~mm}$ whereas the ACD remained unchanged. The authors concluded that flattening of the iris after laser iridotomy for pupillary block causes an increase in the iris-lens contact. The ILCD may be influenced by the presence or absence of an active pupillary block, iris bombe formation, use of pilocarpine, the ambient illumination, and pressure exerted by the plastic cup while performing UBM and this could explain the difference in outcome in the present study.

There was change in the mean central AC depth in PAC but no change in PACG after LPI, which signifies that widening of the central anterior chamber was seen in subacute but not in PACG eyes. No change in ACD was reported by Caronia et $a l^{10}$ and Gazzard et al after $\mathrm{LPI}^{17}$ Marraffa et al ${ }^{19}$ evaluated 30 eyes affected by angleclosure glaucoma that had undergone YAG-laser iridotomy. No significant variation in the central ACD 
was reported, whereas the anterior chamber angle increased from a mean of 10.69 to $21.03^{\circ}$.

Nolan $e t a l^{20}$ gonioscopically evaluated the results of LPI in 164 eyes of 98 subjects. LPI alone failed in 3\% eyes with narrow drainage angles and either peripheral anterior synechiae or raised IOP, but normal optic discs and visual fields. However, in eyes with established glaucomatous optic neuropathy at diagnosis, LPI failed in $47 \%$ of eyes. The authors concluded that although LPI is successful in early stages of PACG, once glaucomatous optic neuropathy associated with synechial angle closure has occurred, iridotomy alone is less effective at controlling IOP.

Kashiwagi et $a l^{21}$ using a newly developed scanning peripheral ACD analyser evaluated the effects of LPI on peripheral ACD in 16 eyes with acute PACG attack, 14 eyes with narrow angle and history of acute attack in their fellow eyes and 13 eyes with chronic angle closure glaucoma. LPI significantly increased the peripheral $\mathrm{ACD}$ and changed the iris contour from convex to flat or concave in all the enrolled eyes. Eyes with narrow angle and history of acute attack in their fellow eyes, that received prophylactic LPI showed the greatest extent increase in $\mathrm{ACD}$, followed by eyes with chronic angle closure glaucoma and eyes with an acute attack of ACG.

The present study highlights that LPI is effective in opening up the angle recess and deepening the anterior chamber in early stages of PACG when there is only an appositional closure of the angle. In such eyes, a peripheral laser iridotomy is an effective therapy as it makes a conduit, allowing aqueous to flow directly from the posterior to the anterior chamber, bypassing the pupillary block. This causes an equalization of the pressure gradient across the iris and allows the iris to flatten and fall posteriorly from the peripheral angle. The end point of these changes in the iris dynamics is a reduction in the appositional obstruction to aqueous outflow caused by the peripheral iris occluding the trabecular meshwork and this helps to prevent intermittent angle closure or an acute attack of PACG in eyes with PAC. The exact mechanism for deepening of the central anterior chamber is not clear. We hypothesize that the obstructed aqueous may find its way into the vitreous and push the lens forward. Decompression of the aqueous with relief of the pupillary block may thus prevent this aberrant aqueous movement, relieve the backward pressure on the lens and result in a deepening of the central anterior chamber.

However, the situation is different in eyes with a chronic angle closure glaucoma with synechial closure of the anterior chamber angle. The presence of dense irido-corneal adhesions in the form of peripheral anterior synechiae, prevents any movement of the peripheral iris and does not permit a widening of the anterior chamber angle in response to the LPI. This is evident from the insignificant change in any of the angle parameters studied on UBM in the present study. Once synechial closure has already occurred, LPI is not beneficial in treating the pathological process and controlling the IOP and thus most PACG patients require medical therapy or surgery after the laser procedure. ${ }^{22}$

In conclusion, this ultrasound biomicroscopic study establishes that LPI opens the narrow angle recess and deepens the anterior chamber in eyes with PAC but is not effective in altering the anterior segment morphology in eyes with PACG. Further studies using UBM or anterior segment optical coherence tomography are required to study the efficacy of LPI in eyes with an acute attack of angle closure glaucoma.

\section{References}

1 Quigley HA. Number of people with glaucoma world wide. Br J Ophthalmol 1996; 80: 389-393.

2 Laser peripheral iridotomy for pupillary-block glaucoma. American Academy of Ophthalmology. Ophthalmology 1994; 101(10): 1749-1758. Review.

3 Go FJ, Akiba Y, Yamamoto T, Kitazawa Y. Argon laser iridotomy and surgical iridectomy in treatment of primary angle closure glaucoma. Jpn J Ophthalmol 1984; 28: 36-46.

4 Rivera AH, Brown RH, Anderson DR. Laser iridotomy vs surgical iridotomy. Have the indications changed? Arch Ophthalmol 1985; 103: 1350-1354.

5 Schwartz LW, Rodrigues MM, Spaeth GL, Streeten B, Douglas C. Argon laser iridotomy in the treatment of patients with primary angle closure or pupillary block glaucoma: a clinicopathologic study. Ophthalmology 1978; 85: 294-309.

6 Fleck BW, Dhillon B, Khanna V, Fairley E, McGlynn C. A randomized, prospective comparison of Nd:YAG laser iridotomy and operative peripheral iridectomy in fellow eyes. Eye 1991; 5: 315-321.

7 Saunders DC. Acute closed angle glaucoma and Nd-YAG laser iridotomy. Br J Ophthalmol 1990; 74: 523-525.

8 Nolan WP, Foster PJ, Devereux JG, Uranchimeg D, Johnson GJ, Baasanhu J. YAG laser iridotomy treatment for primary angle closure in east Asian eyes. Br J Ophthalmol 2000; 84: 1255-1259.

9 McGalliard JN, Wishart PK. The effect of Nd:YAG iridotomy on intraocular pressure in hypertensive eyes with shallow anterior chambers. Eye 1990; 4: 823-829.

10 Caronia RM, Liebmann JM, Stegman Z, Sokol J, Ritch R. Increase in iris-lens contact after laser iridotomy for pupillary block angle closure. Am J Ophthalmol 1996; 122: 53-57.

11 Jin JC, Anderson DR. The effect of iridotomy on iris contour. Am J Ophthalmol 1990; 110: 260-263.

12 Pavlin CJ, Foster FS. Ultrasound biomicroscopy in glaucoma. Acta Ophthalmol Suppl 1992; 204: 7-9.

13 Ishikawa H, Liebmann J, Ritch R. Quantitative assessment of anterior segment using ultrasound biomicroscopy. Curr Opin Ophthalmol 2000; 11: 133-139. 
14 Ishikawa H, Esaki K, Liebmann JM, Uji Y, Ritch R. Ultrasound biomicroscopy dark room provocative testing: a quantitative method for estimating anterior chamber angle width. Jpn J Ophthalmol 1999; 43: 526-534.

15 Sihota R, Dada T, Gupta R, Lakshminarayan P, Pandey RM. Ultrasound biomicroscopy in the subtypes of primary angle closure glaucoma. J Glaucoma 2005; 14(5): 387-391.

16 Sihota R, Sony P, Gupta V, Dada T, Singh R. Comparing glaucomatous optic neuropathy in primary open angle and chronic primary angle closure glaucoma eyes by optical coherence tomography. Ophthalmic Physiol Opt 2005; 25(5): 408-415.

17 Gazzard G, Friedman DS, Devereux JG, Chew P, Seah SKL. A prospective ultrasound biomicroscopy evaluation of changes in anterior segnent morphology after laser iridotomy in Asian eyes. Ophthalmology 2003; 110: 630-638.

18 Yoon KC, Won LD, Cho HJ, Yang KJ. biomicroscopic changes after laser iridotomy or trabeculectomy in angle-closure glaucoma Korean. J Ophthalmol 2004; 18(1): 9-14.

19 Marraffa M, Marchini G, Pagliarusco A, Perfetti S, Toscano A, Brunelli C et al. Ultrasound biomicroscopy and corneal endothelium in Nd:YAG-laser iridotomy. Ophthalmic Surg Lasers 1995; 26(6): 519-523.

20 Nolan WP, Foster PJ, Devereux JG, Uranchimeg D, Johnson GJ, Baasanhu J. YAG laser iridotomy treatment for primary angle closure in east Asian eyes. Br J Ophthalmol 2000; 84(11): 1255-1259.

21 Kashiwagi K, Abe K, Tsukahara S. Quantitative evaluation of changes in anterior segment biometry by peripheral laser iridotomy using newly developed scanning peripheral anterior chamber depth analyser. Br J Ophthalmol 2004; 88(8): 1036-1041.

22 Rosman M, Aung T, Ang LP, Chew PT, Liebmann JM, Ritch R. Chronic angle-closure with glaucomatous damage: longterm clinical course in a North American population and comparison with an Asian population. Ophthalmology 2002; 109(12): 2227-2231. 